\title{
On Boolean posets of numerical events
}

\author{
Dietmar Dorninger ${ }^{1} \cdot$ Helmut Länger ${ }^{1,2}$ (B)
}

Received: 28 March 2021 / Revised: 15 April 2021 / Accepted: 12 May 2021 / Published online: 7 June 2021

(c) The Author(s) 2021

\begin{abstract}
With many physical processes in which quantum mechanical phenomena can occur, it is essential to take into account a decision mechanism based on measurement data. This can be achieved by means of so-called numerical events, which are specified as follows: Let $S$ be a set of states of a physical system and $p(s)$ the probability of the occurrence of an event when the system is in state $s \in S$. A function $p: S \rightarrow[0,1]$ is called a numerical event or alternatively, an $S$-probability. If a set $P$ of $S$-probabilities is ordered by the order of real functions, it becomes a poset which can be considered as a quantum logic. In case the logic $P$ is a Boolean algebra, this will indicate that the underlying physical system is a classical one. The goal of this paper is to study sets of $S$-probabilities which are not far from being Boolean algebras by means of the addition and comparison of functions that occur in these sets. In particular, certain classes of so-called Boolean posets of $S$-probabilities are characterized and related to each other and descriptions based on sets of states are derived.
\end{abstract}

Keywords Quantum effects · Numerical event · Quantum logic · Boolean poset · Set of states

AMS Subject Classification $06 \mathrm{C} 15 \cdot 03 \mathrm{G} 12 \cdot 81 \mathrm{P} 16$

\section{Introduction}

In axiomatic quantum mechanics, orthomodular partially ordered sets and generalizations of them are considered as "quantum logics" that determine the behaviour of a physical system. In particular, if the quantum logic is a Boolean algebra, then one will have reason to assume that one deals with a classical physical system. The elements of a quantum logic can also be interpreted as events, and a Boolean algebra then as the equivalent of a classical field of events as known from probability theory.

Having this in mind, we first recall the notion of a numerical event (cf. Beltrametti and Mạczyński (1991), Beltrametti and Mạczyński (1993) and Mạczyński and Traczyk (1973)).

Helmut Länger

helmut.laenger@tuwien.ac.at

Dietmar Dorninger

dietmar.dorninger@tuwien.ac.at

1 Faculty of Mathematics and Geoinformation, Institute of Discrete Mathematics and Geometry, TU Wien, Wiedner Hauptstraße 8-10, 1040 Vienna, Austria

2 Faculty of Science, Department of Algebra and Geometry, Palacký University Olomouc, 17. listopadu 12, 77146 Olomouc, Czech Republic
Let $S$ be a set of states of a physical system and $p(s)$ the probability of the occurrence of an event, when the system is in state $s \in S$. The function $p$ from $S$ to $[0,1]$ is called a numerical event, or alternatively more precisely an $S$-probability. If ordered by the order $\leq$ of functions and as the case may be endowed with some further properties, a set of $S$-probabilities becomes a partially ordered set (poset) that can be conceived as a quantum logic. In this paper, we study different kinds of such quantum logics, especially those that are not far away from being Boolean algebras. For this end, we provide the following notions.

Let $P$ be a set of $S$-probabilities including the constant functions 0 and 1 , partially ordered by the order of functions. We will call $p, q \in P$ disjoint, in symbols $p \wedge q=0$, if $x \leq p, q$ for $x \in P$ implies $x=0$. Further, $p+q$ and $p-q$ shall denote the sum and difference of $p$ and $q$, respectively, considered as real functions.

Definition 1.1 A set $P$ of $S$-probabilities is called specific if

(1) $0,1 \in P$,

(2) if $p \in P$, then $p^{\prime}:=1-p \in P$,

(3) if $p, q \in P$ and $p \wedge q=0$, then $p+q \in P$. 
Condition (2) seems natural in respect to dealing with probabilities, and as for (3), this condition is motivated by classical fields of events (yet for the time being limited to considering the sum of disjoint events). Conceiving such a field of events as a Boolean ring $R$ of subsets of some set $\Omega$, with + the addition in $R$, one has $A+B=\left(A \cap B^{c}\right) \cup\left(A^{c} \cap B\right)$ for $A, B \in R$, where $\cup$ and $\cap$ stand for the set-theoretic join and meet (i.e. for union and intersection, respectively), ${ }^{c}$ indicates complements in $R$ and $\Omega$ has the role of the unity 1 of $R$. If $A$ and $B$ are disjoint, $A+B=A \cup B \in R$. Further, we observe that due to $R$ having characteristic 2, i.e. + will be the same as,$- 1-A=\Omega-A=\Omega+A=A^{c} \in R$ in coincidence with condition (2).

Two $S$-probabilities $p$ and $q$ are called orthogonal, in symbols $p \perp q$, if $p \leq q^{\prime}$. From condition (3), it follows that $p \wedge q=0$ for $p, q \in P$ implies $p \perp q$. For orthoposets (which specific sets of $S$-probabilities in general are not), this property is known to be Boolean (cf., e.g. Tkadlec (1991); in connection with orthomodular posets see i.a. Mączyński and Traczyk (1973) and Navara and Pták (1989)). We extend this definition to posets $(P, \leq)$ with an antitone involution, i.e. a mapping ' from $P$ to $P$ such that $p \leq q$ implies $p^{\prime} \geq q^{\prime}$ for $p, q \in P$ and $\left(p^{\prime}\right)^{\prime}=p$ for $p \in P$.

Definition 1.2 A poset $P$ with an antitone involution is called Boolean, if $p \wedge q=0$ implies $p \perp q$ for $p, q \in P$.

According to this definition, specific sets of $S$-probabilities are Boolean posets.

Writing $p \vee q$ for the supremum of two elements $p, q$ of a set $P$ of $S$-probabilities and denoting their infimum by $p \wedge q$, we further point out

Remark 1.3 Let $P$ be a set of $S$-probabilities satisfying (1) and (2) and let $p, q \in P$. Then, De Morgan's laws hold in $P$ in the following sense: If $p \vee q$ exists in $P$ then $p^{\prime} \wedge q^{\prime}$ exists in $P$ and $(p \vee q)^{\prime}=p^{\prime} \wedge q^{\prime}$, and if $p \wedge q$ exists in $P$ then $p^{\prime} \vee q^{\prime}$ exists in $P$ and $(p \wedge q)^{\prime}=p^{\prime} \vee q^{\prime}$.

Proof Let $s \in P$. First assume $p \vee q$ to exist in $P$. Then, $(p \vee q)^{\prime} \leq p^{\prime}, q^{\prime}$. If $s \leq p^{\prime}, q^{\prime}$, then $s^{\prime} \geq p, q$ which means $s^{\prime} \geq p \vee q$ from which we infer $s \leq(p \vee q)^{\prime}$. Hence, $p^{\prime} \wedge q^{\prime}$ exists in $P$ and $p^{\prime} \wedge q^{\prime}=(p \vee q)^{\prime}$. The second assertion follows by duality.

Finally, we recall the definitions of two structures of numerical events which we will later relate to specific sets of numerical events.

Definition 1.4 A set $P$ of $S$-probabilities is called a generalized field of events (in short GFE) (cf. Dorninger (2012)), if it satisfies (1), (2) and

(4) if $p, q \in P$ and $p \perp q$ then $p+q \in P$.
If a GFE satisfies

(5) if $p, q, r \in P$ and $p \perp q \perp r \perp p$ then $p+q+r \in P$,

then it is called an algebra of S-probabilities (cf. Beltrametti and Mączyński (1991) and Beltrametti and Mączyński (1993)).

Condition (4) is a special case of condition (5)—just assume $r$ to be 0 .

The goal of this paper is to characterize various classes of specific sets of $S$-probabilities, investigate their interrelations and closeness to Boolean algebras, and indicate when they will actually be Boolean algebras. Moreover, we will consider the question whether (small) sets of $S$-probabilities will belong to a Boolean subalgebra of a specific set of $S$-probabilities and we will characterize specific sets of $S$ probabilities by states.

\section{Specific sets of varying numerical events}

Definition 2.1 An $S$-probability $p$ is called varying, if $p$ is neither $\leq 1 / 2$ nor $\geq 1 / 2$ unless $p=0$ or $p=1$.

The elements of an algebra of $S$-probabilities are varying (cf., e.g. Dorfer et al. (2010)), the elements of GFEs in general are not.

As for data won by experiments: That an $S$-probability is varying often comes up independently or can be achieved by adding further experimental data directed to this purpose.

Now, we will turn our attention to specific sets of $S$ probabilities that are varying. An $S$-probability is called complementary if $p \wedge p^{\prime}=0$ (which by Remark 1.3 is equivalent to $p \vee p^{\prime}=1$ ). A set $P$ of $S$-probabilities with 0 and 1 will be called complemented if all of its elements are complementary. Further, we recall that a poset $P$ with complementation ' which is an antitone involution is called an orthoposet.

Proposition 2.2 A specific set $P$ of varying S-probabilities has the following properties:

(i) $P$ is complemented and hence an orthoposet,

(ii) if $p, q \in P$ and $p \perp q$, then $p \wedge q=0$,

(iii) $P$ is $a$ GFE.

Proof Let $p, q, r \in P$.

(i) If $r \geq p, p^{\prime}$, then $r^{\prime} \leq p \leq r$, from which we infer $r=$ 1 because of $r$ being a varying $S$-probability. Therefore, $p \vee p^{\prime}=1$ and hence, $p \wedge p^{\prime}=0$. 
(ii) If $p \perp q$ and $r \leq p, q$, then because of $p \leq q^{\prime}$, we have $r \leq q, q^{\prime}$ and, since $P$ being complemented, $r=0$ showing $p \wedge q=0$.

(iii) If $p \perp q$, then $p \wedge q=0$ according to (ii), and by condition (3), $p+q \in P$.

Remark 2.3 Let $P$ be a set of $S$-probabilities satisfying (1) and (2). Then, all elements of $P$ are varying if and only if $P$ is complemented.

Proof If all elements of $P$ are varying, then $P$ is complemented according to the proof of Proposition 2.2 (i). Conversely, assume $P$ to be complemented. Let $p \in P$. If $p \leq 1 / 2$, then $p \leq p^{\prime}$ and hence $p=p \wedge p^{\prime}=0$. Dually, if $p \geq 1 / 2$, then $p^{\prime} \leq p$ and we get $p=p \vee p^{\prime}=1$. This proves that every element of $P$ is varying.

An orthoposet that allows a representation by a collection $\Delta$ of subsets of a set $\Omega$ such that

- $\emptyset, \Omega \in \Delta$,

- if $A \in \Delta$ then $\Omega \backslash A \in \Delta$,

- if $A, B \in \Delta$ and $A \cap B=\emptyset$ then $A \cup B \in \Delta$,

is called a concrete logic (cf. Pták (2000)).

Theorem 2.4 The specific sets of varying S-probabilities are exactly the complemented Boolean GFEs. They all are concrete logics.

Proof Let $P$ be a set of $S$-probabilities and $p, q \in P$. First assume $P$ to be a specific set of varying $S$-probabilities. By Proposition 2.2 (i) and (iii), $P$ is a complemented GFE. Conversely, assume $P$ to be a complemented Boolean GFE. Then, as mentioned in Remark 2.3, the elements of $P$ are varying. Further, if $p \wedge q=0$, then $p \perp q$ and thus, $p+q \in P$ according to (4). By Proposition 2.2 (i), specific sets of varying $S$-probabilities are orthoposets and hence, Boolean GFEs are Boolean orthoposets. As mentioned in Tkadlec (1991), Boolean orthoposets are concrete logics due to a proof by Navara and Pták about Boolean orthomodular posets which does not make use of orthomodularity (cf. Navara and Pták (1989)).

Since any specific set of varying $S$-probabilities is a concrete logic, its elements can be represented by functions which have only the values 0 or 1 . So, these $S$-probabilities must be varying from the outset. If $S$ is finite, Theorem 2.4 leads to the conclusion that the specific sets of varying $S$-probabilities are Boolean algebras, since finite Boolean orthoposets are Boolean algebras. So to distinguish between a classical and a quantum mechanical behaviour by measurements in the form of numerical events, one would need data from $S$-probabilities for a continuous set $S$ of states.
Next, we turn our attention towards the connection of specific sets of varying $S$-probabilities and algebras of $S$-probabilities.

Lemma 2.5 The complemented Boolean GFEs are exactly the algebras of S-probabilities that are Boolean.

Proof According to Theorem 2.4, a complemented Boolean GFE is a concrete logic, and that such a GFE is an algebra of $S$-probabilities was already shown in Dorninger (2012). Conversely, every algebra of $S$-probabilities that is Boolean is also a Boolean GFE, and an arbitrary algebra of $S$ probabilities is complemented (because it is an orthoposet, first ascertained in Mączyński and Traczyk (1973)).

In fact, algebras of $S$-probabilities are orthomodular posets with a full set of states, and vice versa (cf. Mączyński and Traczyk (1973)). Further, an orthomodular poset is Boolean if and only if it is infimum faithful (cf. Godowski (1980)). To be infimum faithful means that $p \wedge q$ exists if and only if $p$ and $q$ commute, i.e. $p=(p \wedge q) \vee\left(p \wedge q^{\prime}\right)$. Since denoting an algebra of $S$-probabilities $P$ as Boolean could be mixed up with $P$ being a Boolean algebra, what in general is not the case, we rather prefer the notion infimum faithful. In the light of Theorem 2.4 and Lemma 2.5, we then obtain

Theorem 2.6 The specific sets of varying S-probabilities are exactly the infimum faithful algebras of S-probabilities.

Returning to the motivation of the definition of specific sets of $S$-probabilities by Boolean rings, in line with Theorems 2.4 and 2.6, we can now remark:

Remark 2.7 An infimum faithful algebra of $S$-probabilities which is a Boolean algebra can be conceived as a Boolean ring if one extends + to arbitrary $S$-probabilities $p$ and $q$ by assuming within the pointwise addition of the functions $p$ and $q$ that $1+1=0$, and taking $p \cdot q:=p \wedge q$ for the ring's multiplication.

\section{Further classes of specific sets of $S$-probabilities}

Let $P$ be a set of $S$-probabilities. We consider the following conditions:

(6) If $p, q \in P$ and $p \wedge q=0$ then $p+q=p \vee q \in P$,

(7) if $p, q, r \in P, p \perp q \perp r$ and $p \wedge r=0$ then $p+q+r \in$ $P$,

(8) if $p, q, r \in P, p \perp q \perp r$ and $p \wedge r=0$ then $p+q+r \leq$ 1 .

Condition (6) can be motivated by regarding a classical field of events as a Boolean ring $R$ for which it is the case 
that $A \cap B=\emptyset$ for $A, B \in R$ implies $A+B=A \cup B$ (see Introduction). For short, we will denote specific sets of $S$-probabilities that satisfy condition (6) as $\vee$-specific (joinspecific) sets of $S$-probabilities. If (1), (2) and (7) hold, $P$ is called a structured set of $S$-probabilities (cf. Dorninger and Länger (2016)), and if (1), (2) and (8) are satisfied $P$ is known as a weakly structured set of $S$-probabilities (cf. Dorninger and Länger (2016)).

Now, we define the following classes of sets of $S$ probabilities:

$\mathcal{C}_{1}$ : class of specific sets of $S$-probabilities,

$\mathcal{C}_{2}$ : class of $\vee$-specific sets of $S$-probabilities (satisfying (6)),

$\mathcal{C}_{3}$ : class of structured sets of $S$-probabilities (for which

(7) is distinctive),

$\mathcal{C}_{4}$ : class of weakly structured sets of $S$-probabilities (characterized by (8)).

$\mathcal{C}_{2}$ is a subclass of $\mathcal{C}_{1}$, and this is also true for $\mathcal{C}_{3}$ as one can see by setting $q=0$ within (7).

Lemma 3.1 We have $\mathcal{C}_{3} \subseteq \mathcal{C}_{2} \subseteq \mathcal{C}_{4}$.

Proof Let $P$ be a set of $S$-probabilities and $p, q, r \in P$. First assume $P \in \mathcal{C}_{3}$. As already mentioned above, $P$ is a specific set of $S$-probabilities. If $p \wedge q=0$, then $p+q=p \vee q$ because for $r \geq p$, we have $p \perp r^{\prime} \perp q$ besides $p \wedge q=0$ from which we can conclude that $p+r^{\prime}+q \in P$ showing that $p+q \leq r$ and hence, $p+q=p \vee q$ which explains that $P \in \mathcal{C}_{2}$ and hence, $\mathcal{C}_{3} \subseteq \mathcal{C}_{2}$. Now, assume $P \in \mathcal{C}_{2}$, $p \perp q \perp r$ and $p \wedge r=0$. Since $p \leq q^{\prime}$ and also $r \leq q^{\prime}$, we obtain that $p+r=p \vee r \leq q^{\prime}$ from which we infer $p+q+r \leq 1$. Therefore, $P \in \mathcal{C}_{4}$ and hence, $\mathcal{C}_{2} \subseteq \mathcal{C}_{4}$.

Lemma 3.2 We have $\mathcal{C}_{2}=\mathcal{C}_{1} \cap \mathcal{C}_{4}$.

Proof Let $P$ be a specific set of $S$-probabilities which is also a weakly structured set of $S$-probabilities and assume $p, q, r \in$ $P$ such that $p \wedge q=0$ and $r \geq p, q$. Then, $p \perp r^{\prime} \perp q$ and hence, $p+r^{\prime}+q \leq 1$, i.e. $p+q \leq r$ which shows $p+q=p \vee q$. Since according to Lemma $3.1 \mathcal{C}_{2} \subseteq \mathcal{C}_{4}$, we are done.

Theorem 3.3 The class of structured sets of S-probabilities is a proper subclass of the class of $\vee$-specific sets of $S$ probabilities which on its part is a proper subclass of the class of weakly structured sets of S-probabilities unless one assumes that only specific sets of S-probabilities are taken into account.

Proof As for the inclusions to be proper, in agreement with Lemma 3.1, it suffices to consider the following two examples:
First, assume $|S|=2$ and define

$P:=\{(0,0),(1 / 4,1 / 4),(1 / 4,3 / 4),(3 / 4,1 / 4)$,

$(3 / 4,3 / 4),(1,1)\}$.

Then, $P \in \mathcal{C}_{2}$, but $P \notin \mathcal{C}_{3}$ since

$$
\begin{aligned}
& (0,0) \perp(1 / 4,1 / 4) \perp(1 / 4,3 / 4) \text { and } \\
& (0,0) \wedge(1 / 4,3 / 4)=(0,0),
\end{aligned}
$$

but

$(0,0)+(1 / 4,1 / 4)+(1 / 4,3 / 4)=(1 / 2,1) \notin P$.

Second example: Again we assume $|S|=2$ and this time define

$P:=\{(0,0),(0,1 / 2),(1 / 2,0),(1 / 2,1),(1,1 / 2),(1,1)\}$.

Then, $P \in \mathcal{C}_{4}$, but $P \notin \mathcal{C}_{2}$ since

$$
\begin{aligned}
& (0,1 / 2) \wedge(1 / 2,0)=(0,0), \text { but } \\
& (0,1 / 2)+(1 / 2,0)=(1 / 2,1 / 2) \notin P .
\end{aligned}
$$

That the $\vee$-specific sets of $S$-probabilities are exactly the elements of $\mathcal{C}_{1} \cap \mathcal{C}_{4}$ is confirmed by Lemma 3.2.

Next we will discuss the question how far $\vee$-specific sets of $S$-probabilities are away from being Boolean algebras. A first reference to this can be a subclass of $\mathcal{C}_{3}$.

Theorem 3.4 (cf. Dorninger and Länger (2016)) The complemented members of the class $\mathcal{C}_{3}$ of structured sets of $S$-probabilities are exactly the infimum faithful algebras of S-probabilities.

Though $\mathcal{C}_{3}$ is a proper subclass of $\mathcal{C}_{2}$, more incisive properties have to be taken into account to distinguish $\mathcal{C}_{2}$ from Boolean algebras: e.g. if a complemented structured set of $S$-probabilities $P$ is finite, it is a Boolean algebra, because, as already mentioned, finite Boolean orthoposets are Boolean algebras. Further, $P$ is a Boolean algebra if it is orthocomplete (cf. Tkadlec (1994)). (To be orthocomplete means that the supremum of any set of pairwise orthogonal elements of $P$ has to belong to $P$.) Moreover, if $P$ is a lattice (i.e. $p \vee q$ and $p \wedge q$ exist for all $p, q \in P$ ), then we also have a Boolean algebra (cf. Tkadlec (1991)). That $P$ is lattice-ordered can be characterized by a simple criterion: According to Theorem 2.4, $P$ is a concrete logic, and as shown in Dorninger and Länger (2016), a structured set of $S$-probabilities $P$ which is a concrete logic is a lattice if and only if for all $p, q \in P$ $\max (p, q) \in P$ (the maximum of the functions considered pointwise). 
There are many papers in which (arbitrary) classes of algebras of $S$-probabilities are characterized to be Boolean algebras by specifying some structural properties-for an overview of these papers see Dorninger (2020) — and there are numerous results on Boolean orthoposets and concrete logics which can all be applied to fathom the distance between specific sets of $S$-probabilities and Boolean algebras (cf. i.a. Klukowski (1975), Navara and Pták (1989), Pták (2000), Tkadlec (1991), Tkadlec (1994) and Tkadlec (1997)).

Sometimes it is not of interest if a whole algebra of $S$ probabilities $P$ is a Boolean algebra but if a (usually small) subset of $P$ belongs to a Boolean subalgebra of $P$. If this were the case, this would indicate that one locally deals with a classical physical system. To answer this question, the existence of some further $S$-probabilities in $P$ will have to be asked for, but the knowledge of $P$ in detail will not be important.

So let us assume that a subset $\left\{p_{1}, \ldots, p_{n}\right\}$ of a known or hypothetically assumed infimum faithful algebra of $S$ probabilities $P$ is given. If $p_{1}, \ldots, p_{n}$ are pairwise orthogonal, then there does exist a Boolean subalgebra of $P$ wherein $p_{1}, \ldots, p_{n}$ are contained, as it is well known for every subset of mutually orthogonal elements of an orthomodular poset (cf. Dorninger et al. (2020)), and as proved in Mączyński and Traczyk (1973), every algebra of $S$-probabilities is orthomodular. So, let us suppose that $\left\{p_{1}, \ldots, p_{n}\right\}$ is an arbitrary subset of $P$.

Having in mind that the elements of $P$ can only assume the values 0 and 1 (cf. Theorems 2.4 and 2.6) and defining $p \cdot q$ for $p, q \in P$ by $(p \cdot q)(s)=p(s) \cdot q(s)$ for $s \in S$, one obtains that if $p \cdot q$ exists in $P$, then $p \cdot q=p \wedge q$. It is obvious then that $p^{k}=p$ for $k=1,2,3, \ldots$ and that the multiplication is associative.

In Section 2, we have defined what it means that $p$ and $q$ commute. We will express this fact by writing $p \mathrm{C} q$ and point out that for orthomodular posets, $p \mathrm{C} q$ is equivalent to $q \mathrm{C} p$. Further, we agree to write $\bigwedge B$ for the infimum of the elements of a finite subset $B$ of $P$. Now, we can prove the following

Theorem 3.5 The set $\left\{p_{1}, \ldots, p_{n}\right\}$ is contained in a Boolean subalgebra of an infimum faithful algebra of $S$-probabilities $P$ if and only if $p_{i_{1}} \cdots p_{i_{n}} \in P$ for all $i_{1}, \ldots, i_{n} \in$ $\{1, \ldots, n\}$.

Proof Assume $n=2$. Then according to Theorem 3.4, in Dorninger et al. (2020), $\left\{p_{1}, p_{2}\right\}$ is contained in a Boolean subalgebra of $P$ if and only if $p_{1} \wedge p_{2}\left(:=\min \left(p_{1}, p_{2}\right)\right) \in P$ which in our notion means that $p_{1} \cdot p_{2} \in P$. In this theorem, it is also stated that $p_{1} \bar{\wedge} p_{2}\left(=p_{1} \cdot p_{2}\right) \in P$ is equivalent to $p_{1} \mathrm{C} p_{2}$.

Next we make use of Corollary 2.3 in Dorninger and Länger (2014) which says: Let $A$ be a subset of an orthomodular poset $P$ with $n>1$ elements. Then, $A$ is contained in an Boolean subalgebra of $P$ if and only if $(\bigwedge B) \mathrm{C}(\bigwedge D)$ for every $k \in\{1, \ldots, n-1\}$ and every $k$-element subsets $B$ and $D$ of $A$.

Now, we assume $P$ to be our structured set of $S$ probabilities and $A=\left\{p_{1}, \ldots, p_{n}\right\} \subseteq P$. Then, $\bigwedge B$ and $\bigwedge D$ are the products $p_{B}$ and $p_{D}$ of the elements of $B$ and $D$, respectively. If $p_{i_{1}} \cdots p_{i_{n}} \in P$ for all $i_{1}, \ldots, i_{n} \in$ $\{1, \ldots, n\}$, then $p_{B} \mathrm{C} p_{D}$ for every subsets $B$ and $D$ of $A$ with $k \leq n-1$ elements since $p_{B} \cdot p_{D}$ is an element of $P$ and, as mentioned above, $p_{B} \mathrm{C} p_{D}$ is equivalent to $p_{B} \cdot p_{D} \in P$. Thus, we can conclude that the elements of $A$ are contained in a Boolean subalgebra of $P$. The converse is obvious.

Besides the possibility to describe sets of $S$-probabilities by structural properties, one can also try to characterize them by states, as was done by M. J. Mạczyński and T. Traczyk, who characterized algebras of $S$-probabilities as the orthomodular posets which have a full set of states (cf. Mạczyński and Traczyk (1973)).

\section{Algebraic representations of specific sets of S-probabilities}

We begin by extending the commonly known notion of a state to the class of bounded posets $P$ with an antitone involution.

Definition 4.1 A specific state on a bounded poset $P=$ $\left(P, \leq,{ }^{\prime}, 0,1\right)$ with an antitone involution is a mapping $s$ from $P$ to $[0,1]$ satisfying the following conditions for all $p, q \in P$ :

(S1) $s(0)=0$ and $s(1)=1$,

(S2) $s\left(p^{\prime}\right)=1-s(p)$,

(S3) if $p \leq q$, then $s(p) \leq s(q)$,

(S4) if $p \wedge q=0$, then there exists some $r \in P$ with $r \geq p, q$ and $s(r)=s(p)+s(q)$.

If for $p, q \in P$ with $p \wedge q=0$ the element $p \vee q$ exists in $P$, then a specific state on $P$ satisfying

(S5) if $p, q \in P$ and $p \wedge q=0$ then $s(p \vee q)=s(p)+s(q)$,

is called a pseudostate on $P$ (cf. Dorninger and Länger (2016)).

A set $T$ of specific states on $P$ is called full if for $p, q \in P$, $s(p) \leq s(q)$ for all $s \in T$ implies $p \leq q$, and a set $T$ of specific states on $P$ is called uniform if for disjoint $p, q \in P$ condition (S4) is satisfied for all $s \in T$ with the very same $r$. (With pseudostates, one can take $r=p \vee q$.)

Theorem 4.2 Up to isomorphism, the specific sets of $S$ probabilities are exactly the bounded posets with an antitone involution having a full and uniform set of specific states. 
Proof Let $P=\left(P, \leq,^{\prime}, 0,1\right) \in \mathcal{C}_{1}$ with $P \subseteq[0,1]^{S}, a \in S$ and $p, q \in P$. Then, clearly, $P$ is a bounded poset with an antitone involution. We define $s_{x}(r):=r(x)$ for all $x \in S$ and $r \in P$. Then, we have

(S1) $s_{a}(0)=0(a)=0$ and $s_{a}(1)=1(a)=1$,

(S2) $s_{a}\left(p^{\prime}\right)=p^{\prime}(a)=1-p(a)=1-s_{a}(p)$,

(S3) if $p \leq q$ then $s_{a}(p)=p(a) \leq q(a)=s_{a}(q)$,

(S4) if $p \wedge q=0$ then $p+q \in P, p+q \geq p, q$ and $s_{a}(p+$ $q)=(p+q)(a)=p(a)+q(a)=s_{a}(p)+s_{a}(q)$.

Further, if $s_{x}(p) \leq s_{x}(q)$ for all $x \in S$, then $p \leq q$. Hence, $\left\{s_{x} \mid x \in S\right\}$ is a full and uniform set of specific states on $P$.

Conversely, let $P=\left(P, \leq,{ }^{\prime}, 0,1\right)$ be a bounded poset with an antitone involution which has a full and uniform set $S$ of specific states and let $p, q, r \in P$ and $s \in S$. We define $(f(u))(t):=t(u)$ for all $u \in P$ and $t \in S$. Then, the following assertions are equivalent: $f(p) \leq f(q)$, $(f(p))(t) \leq(f(q))(t)$ for all $t \in S, t(p) \leq t(q)$ for all $t \in S, p \leq q$. Therefore $f(p)=f(q)$ if and only if $p=q$. Next we will prove $f(P) \in \mathcal{C}_{1}$ :

(1) $(f(0))(s)=s(0)=0$ and $(f(1))(s)=s(1)=1$; thus, $0=f(0) \in f(P)$ and $1=f(1) \in f(P)$.

(2) $\left(f\left(p^{\prime}\right)\right)(s)=s\left(p^{\prime}\right)=1-s(p)=1-(f(p))(s)=$ $(f(p))^{\prime}(s)$; therefore, $(f(p))^{\prime}=f\left(p^{\prime}\right) \in f(P)$.

(3) Assume $f(p) \wedge f(q)=f(0)$. If $r \leq p, q$, then $f(r) \leq$ $f(p), f(q)$, from which we infer $f(r)=f(0)$, i.e. $r=0$, showing $p \wedge q=0$. Accordingly, there exists some $u \in P$ (which is independent of $s$ ) with $s(u)=$ $s(p)+s(q)$. Now,

$$
\begin{aligned}
& (f(p)+f(q))(s)=(f(p))(s)+(f(q))(s) \\
& \quad=s(p)+s(q)=s(u)=(f(u))(s), \\
& \text { i.e. } f(p)+f(q)=f(u) \in f(P) .
\end{aligned}
$$

From this, we can conclude that $f(P)=\left(f(P), \leq,{ }^{\prime}, 0,1\right) \in$ $\mathcal{C}_{1}$ and that $f$ is an isomorphism from $P$ onto $f(P)$. Hence, $P$ is isomorphic to a member of $\mathcal{C}_{1}$.

As shown in Dorninger and Länger (2016) up to isomorphism, the weakly structured sets of $S$-probabilities are exactly the bounded posets with an antitone involution in which the join of two disjoint elements exists and which have a full set of pseudostates, which in the light of Lemma 3.2 then reads

Theorem 4.3 Up to isomorphism, the $\vee$-specific sets of $S$ probabilities are exactly the bounded posets with an antitone involution in which the sum of two disjoint elements equals their join and which have a full set of pseudostates.
Theorems 4.2 and 4.3 are analogues to the theorem mentioned above that up to isomorphism, the algebras of $S$-probabilities are exactly the orthomodular posets having a full set of states.

\section{Conclusions}

To find out whether quantum mechanical effects can occur in a physical or technical system, various classes of sets of numerical events were studied, i.e. data-sets obtained by surveying the system which then form or contribute to the underlying quantum logic. In particular, sets of numerical events, called specific sets, were analyzed that are located at the threshold between classical and quantum behavior, a situation that can be characterized by the fact that in case of a classical physical system the underlying logic is a Boolean algebra. The interrelations of specific sets of numerical events and their closeness to Boolean algebras were ascertained and the question was answered when an unstructured set of numerical events can be a part of a Boolean algebra. Moreover, specific sets of numerical events were characterized by the states they admit.

Author Contributions The authors contributed equally to this manuscript, both authors have read and agreed to the published version of the manuscript.

Funding Open access funding provided by Austrian Science Fund (FWF).

Data availability Not applicable.

Code availability Not applicable

\section{Declarations}

Conflict of interests There are no conflicts of interest.

Ethical standards This article does not contain any studies with human participants or animals performed by any of the authors.

Open Access This article is licensed under a Creative Commons Attribution 4.0 International License, which permits use, sharing, adaptation, distribution and reproduction in any medium or format, as long as you give appropriate credit to the original author(s) and the source, provide a link to the Creative Commons licence, and indicate if changes were made. The images or other third party material in this article are included in the article's Creative Commons licence, unless indicated otherwise in a credit line to the material. If material is not included in the article's Creative Commons licence and your intended use is not permitted by statutory regulation or exceeds the permitted use, you will need to obtain permission directly from the copyright holder. To view a copy of this licence, visit http://creativecomm ons.org/licenses/by/4.0/. 


\section{References}

Beltrametti EG, Mạczyński MJ (1991) On a characterization of classical and nonclassical probabilities. J Math Phys 32:1280-1286

Beltrametti EG, Mączyński MJ (1993) On the characterization of probabilities: a generalization of Bell's inequalities. J Math Phys 34:4919-4929

Dorfer G, Dorninger D, Länger H (2010) On the structure of numerical event spaces. Kybernetica 46:971-981

Dorninger D (2012) On the structure of generalized fields of events. Contributions General Algebra 20:29-34

Dorninger D (2020) Identifying quantum logics by numerical events. Math Slovaca 70:41-50

Dorninger D, Länger H (2014) A note of Boolean subsets of orthomodular posets. Ital J Pure Appl Math 32:277-282

Dorninger D, Länger H (2016) On bounded posets arising from quantum mechanical measurements. Int J Theor Phys 55:4453-4461

Dorninger D, Länger H, Mączyński MJ (2020) Boolean properties and Bell-like inequalities of numerical events. Rep Math Phys 85:147162

Finch PD (1969) On the lattice structure of quantum logic. Bull Aust Math Soc 1:333-340
Godowski RM (1980) Commutativity in orthomodular posets. Rep Math Phys 18:347-351

Klukowski J (1975) On the representation of Boolean orthomodular partially ordered sets. Demonstratio Math 8:405-423

Mackey GW (2004) Mathematical foundations of quantum mechanics. Dover, Mineola

Mạczyński MJ, Traczyk T (1973) A characterization of orthomodular partially ordered sets admitting a full set of states. Bull Acad Polon Sci Sér Sci Math Astronom Phys 21:3-8

Navara M, Pták P (1989) Almost Boolean orthomodular posets. J Pure Appl Algebra 60:105-111

Pták P (2000) Concrete quantum logics. Int J Theor Phys 39:827-837

Tkadlec J (1991) A note on distributivity in orthoposets. Demonstratio Math 24:343-346

Tkadlec J (1994) Boolean orthoposets - concreteness and orthocompleteness. Math Bohem 119:123-128

Tkadlec J (1997) Conditions that force an orthomodular poset to be a Boolean algebra. Tatra Mt Math Publ 10:55-62 